\title{
Post-translational Modifications in Oral Bacteria and Their Functional Impact
}

\author{
Qizhao Ma ${ }^{1,2+}$, Qiong Zhang ${ }^{1,2+}$, Yang Chen ${ }^{1,2}$, Shuxing Yu ${ }^{1,2}$, Jun Huang ${ }^{1,2}$, Yaqi Liu ${ }^{1,2}$, \\ Tao Gong ${ }^{1}$, Yuqing $\mathrm{Li}^{1 *}$ and Jing Zou ${ }^{1,2 *}$ \\ ' State Key Laboratory of Oral Diseases, National Clinical Research Center for Oral Diseases, West China Hospital \\ of Stomatology, Sichuan University, Chengdu, China, ${ }^{2}$ Department of Pediatric Dentistry, West China Hospital \\ of Stomatology, Sichuan University, Chengdu, China
}

Oral bacteria colonize the oral cavity, surrounding complex and variable environments. Post-translational modifications (PTMs) are an efficient biochemical mechanism across

OPEN ACCESS

Edited by:

Valerie Carabetta,

Cooper Medical School of Rowan University, United States

Reviewed by:

Saori Kosono,

The University of Tokyo, Japan

Saswat S. Mohapatra,

Berhampur University, India

*Correspondence:

Yuqing $\mathrm{Li}$

liyuqing@scu.edu.cn

Jing Zou

zoujing@scu.edu.cn

${ }^{t}$ These authors have contributed equally to this work

Specialty section:

This article was submitted to Microbial Physiology and Metabolism, a section of the journal

Frontiers in Microbiology

Received: 28 September 2021 Accepted: 02 November 2021 Published: 02 December 2021

Citation:

Ma Q, Zhang Q, Chen Y, Yu S,

Huang J, Liu Y, Gong T, Li Y and

Zou J (2021) Post-translational

Modifications in Oral Bacteria

and Their Functional Impact.

Front. Microbiol. 12:784923.

doi: 10.3389/fmicb.2021.784923 all domains of life. Oral bacteria could depend on PTMs to quickly regulate their metabolic processes in the face of external stimuli. In recent years, thanks to advances in enrichment strategies, the number and variety of PTMs that have been identified and characterized in oral bacteria have increased. PTMs, covalently modified by diverse enzymes, occur in amino acid residues of the target substrate, altering the functions of proteins involved in different biological processes. For example, Ptk1 reciprocally phosphorylates Php1 on tyrosine residues 159 and 161, required for Porphyromonas gingivalis EPS production and community development with the antecedent oral biofilm constituent Streptococcus gordonii, and in turn Php1 dephosphorylates Ptk1 and rapidly causes the conversion of Ptk1 to a state of low tyrosine phosphorylation. Protein acetylation is also widespread in oral bacteria. In the acetylome of Streptococcus mutans, 973 acetylation sites were identified in 445 proteins, accounting for $22.7 \%$ of overall proteins involving virulence factors and pathogenic processes. Other PTMs in oral bacteria include serine or threonine glycosylation in Cnm involving intracerebral hemorrhage, arginine citrullination in peptidylarginine deiminases (PADs), leading to inflammation, lysine succinylation in $P$. gingivalis virulence factors (gingipains, fimbriae, RagB, and PorR), and cysteine glutathionylation in thioredoxin-like protein (Tlp) in response to oxidative stress in S. mutans. Here we review oral bacterial PTMs, focusing on acetylation, phosphorylation, glycosylation, citrullination, succinylation, and glutathionylation, and corresponding modifying enzymes. We describe different PTMs in association with some examples, discussing their potential role and function in oral bacteria physiological processes and regulatory networks. Identification and characterization of PTMs not only contribute to understanding their role in oral bacterial virulence, adaption, and resistance but will open new avenues to treat oral infectious diseases.

Keywords: post-translational modifications, bacteria, physiology, bacterial virulence, Streptococcus mutans, Porphyromonas gingivalis 


\section{INTRODUCTION}

Post-translational modifications (PTMs) expand nature's inventory of the 20 proteinogenic amino acids by inducing covalent modifications in amino acid residues (BeckSickinger and Mörl, 2006). These modifications alter protein functions, such as enzymatic activity, localization, stability, or interactions with other molecules across all domains of life. As the main component of oral microflora, oral bacteria depend on PTMs to quickly respond to external stimuli and regulate their physiological processes. In oral bacterial species, common modifications include phosphorylation, acetylation, glycosylation, citrullination, succinylation, and glutathionylation (Wu et al., 2019; Zeng et al., 2020).

Post-translational modifications modulate bacterial physiological processes and regulate bacterial adaptability to the environment. The complex and harsh oral environment connects the internal body with the external environment. Due to the particularity of the oral anatomy, the oral bacteria are susceptible to host conditions, e.g., dietary habits, immune responses, $\mathrm{pH}$, poor oral hygiene, oxygen tension, and lifestyle. When oral bacteria encounter rapidly changing and hostile environmental stresses, PTMs are a highly efficient mechanism to regulate protein function (e.g., from inactive to active) compared with the energy-extensive processes of gene expression, protein synthesis, and protein degradation. For example, sensor histidine kinase is autophosphorylated in two-component regulatory systems (TCS), quickly activating the downstream response regulator (RR). Such "fast-switching" events allow oral bacteria to quickly respond to external stimuli, which is more efficient than the regulation of gene expression. Under oxidative stress, proteins could use $S$-glutathionylation to maintain intracellular redox homeostasis (Loi et al., 2015).

In addition, PTMs play an essential role in regulating bacterial virulence, subsequently affecting oral homeostasis and disease conditions. For example, oral bacteria facilitate bacterial colonization and the accumulation of multicellular clusters by glucosyltransferases to synthesize extracellular polysaccharides (EPS), the major constituents of the plaque biofilm. Notably, the acetylation level of glucosyltransferases is closely associated with their activity. In addition to local influences, pathogenic bacteria in the oral cavity can gain access to the bloodstream, causing systemic infections by secreting virulence factors (Liu et al., 2012; Blod et al., 2018; Lira-Junior and Boström, 2018; Philip et al., 2018; Reddy et al., 2018; Saikaly et al., 2018). Multiple PTMs occur in these secreted virulence factors. For example, the peptidylarginine deiminase (PAD) secreted by Porphyromonas gingivalis not only could citrullinate argininespecific protease A (RgpA) and arginine-specific protease B (RgpB), the important virulence of periodontitis but citrullinate human host proteins that are closely related to systemic diseases. The glycosylation of serine-rich repeat (SRR) glycoprotein gordonii surface protein (GspB) in Streptococcus gordonii contributes to the infective endocarditis (IE) by binding to human platelets (Seepersaud et al., 2020).

Unlike mRNA or protein synthesis, PTMs are not templated but usually rely on recognition and modification by the relevant enzyme. Most PTMs are reversible and dynamic, i.e., modified chemical groups can be placed onto or removed from the polypeptide chain by specialized enzymes, such as the kinases Stk1 and phosphatases Stp1 in Streptococcus agalactiae and acetyltransferases Pat and deacetylase CobB in $P$ gingivalis. In addition to the specialized enzymes, some chemical compounds are also involved in protein modifications. For example, acetylcoenzyme A (Ac-CoA) directly increased the acetylation of RprY in P. gingivalis. Succinylation was also mediated nonenzymatically with succinyl-CoA as the donor (Wagner and Payne, 2013; Wagner and Hirschey, 2014).

Moreover, proteins commonly carry multiple modifications, and some residues can simultaneously carry several modifications. For instance, acetylation and succinylation in P. gingivalis are extensively overlapped. RprY could be both acetylated and phosphorylated. Such PTM crosstalk can mediate complementary or opposing effects, resulting in a complex combination of the structure and function of the target protein, which illustrates the complexity and flexibility of the underlying regulatory processes.

With the development of novel enrichment strategies of PTM sites in past decades, new PTMs are constantly being discovered, with new targets and sites of known PTM in oral bacteria. Consequently, research into PTMs is burgeoning and growing, particularly in regulating bacterial physiological processes (Walsh et al., 2005; Cain et al., 2014). This review introduces the significant types of PTMs in oral bacteria and discusses their roles in bacterial physiological and pathological processes (Table 1).

\section{MAJOR PTMS IN ORAL BACTERIA AND THEIR FUNCTIONAL IMPACT}

\section{Protein Phosphorylation}

Common protein phosphorylation involves kinase-catalyzed addition of phosphate to amino acid side chains of histidine (His), aspartate (Asp), serine (Ser), threonine (Thr), and tyrosine (Tyr) in oral bacteria. Protein phosphorylation can be readily reversed by phosphatase-mediated hydrolysis (dephosphorylation) to regenerate unmodified hydroxyls, i.e., changing the residue from hydrophobic to hydrophilic (Mijakovic et al., 2016). Therefore, reversible protein phosphorylation can regulate the characteristics of proteins rapidly and react quickly to external stimuli. There are many donor sources of phosphate groups, the most utilized of which are active phosphate groups and adenosine triphosphate (ATP). Most phosphorylated side chains or kinases are residue-specific, and different phosphorylated amino acid side chains have different chemical stability and distinct impacts on hemostasis and quality control. Protein phosphorylation is widely involved in metabolic processes, including cell survival, virulence factor, and environmental adaptation in oral bacteria. Different phosphorylation modifications will be reviewed using the families of kinases and the residues they phosphorylate in this part (Figure 1).

\section{Histidine Phosphorylation}

The best-characterized histidine phosphorylation in oral bacteria belongs to the so-called two-component systems (TCSs), consisting of a membrane-located sensor His kinase (HK) and 
TABLE 1 | Post-translational modifications (PTMs) and their functional roles in oral bacteria.

\begin{tabular}{|c|c|c|c|c|c|}
\hline PTM & Protein & Target residue & Function & Organism & References \\
\hline \multicolumn{6}{|l|}{ Protein phosphorylation } \\
\hline \multirow[t]{7}{*}{ Histidine phosphorylation } & Vick & His residue & $\begin{array}{l}\text { VicR, regulating the expression of } \\
g t f B / C\end{array}$ & S. mutans & Wang et al., 2021 \\
\hline & ComD & His residue & $\begin{array}{l}\text { ComE, inducing bacterial competence } \\
\text { and exogenous DNA uptake }\end{array}$ & S. mutans & Shepherd et al., 2012 \\
\hline & NsrS & His residue & NsrR, regulating the expression of $n s r X$ & S. mutans & $\begin{array}{l}\text { Kawada-Matsuo et al., } \\
2013\end{array}$ \\
\hline & LcrS & His residue & $\begin{array}{l}\text { LcrR, regulating the expression of } \\
\text { IctFEG }\end{array}$ & S. mutans & $\begin{array}{l}\text { Kawada-Matsuo et al., } \\
2013\end{array}$ \\
\hline & Haes & His226 & $\begin{array}{l}\text { HaeR, regulating iron } \\
\text { uptake/acquisition genes }\end{array}$ & P. gingivalis & $\begin{array}{l}\text { Huynh et al., 2010; } \\
\text { Willett and Kirby, } 2012\end{array}$ \\
\hline & PorY & His residue & PorX, type IX secretion system & P. gingivalis & Kadowaki et al., 2016 \\
\hline & Fims & His residue & FimR, regulating the expression of fimA & P. gingivalis & Nishikawa et al., 2004 \\
\hline \multirow{4}{*}{$\begin{array}{l}\text { Serine/threonine } \\
\text { phosphorylation }\end{array}$} & CovR & Thr65 & Regulate expression of CAMP factor & S. agalactiae & Lin et al., 2009 \\
\hline & PknB & $\begin{array}{l}\text { Ser and/or Thr } \\
\text { residue }\end{array}$ & $\begin{array}{l}\text { Regulating the activity of PppL, VicKR, } \\
\text { and ComDE }\end{array}$ & S. mutans & $\begin{array}{l}\text { Banu et al., 2010; Zhu } \\
\text { and Kreth, } 2010\end{array}$ \\
\hline & SpxB & Thr409, 415, 508 & Mediating pyruvate conversion & S. sanguinis & Mu et al., 2021 \\
\hline & $\begin{array}{c}\text { PGN_0375, PGN_0500, } \\
\text { PGN_0724, PGN_0733, } \\
\text { PGN_0880 }\end{array}$ & Ser and/or Thr & Not determined & P. gingivalis & Izumigawa et al., 2016 \\
\hline \multirow[t]{3}{*}{ Tyrosine phosphorylation } & Php1 & Tyr159, 166 & $\begin{array}{l}\text { Exopolysaccharide production, } \\
\text { proteinases phosphorylation }\end{array}$ & $P$. gingivalis & Jung et al., 2019 \\
\hline & RprY & Tyr41 & Type IX secretion system & P. gingivalis & Shen et al., 2020 \\
\hline & SpxB & Tyr409, 415, 588 & $\begin{array}{l}\text { Intraspecies and interspecies } \\
\text { competition }\end{array}$ & S. sanguinis & Mu et al., 2021 \\
\hline \multirow[t]{3}{*}{ Protein acetylation } & RgpA, RgpB, Kgp & Lysine residue & Activation/maturation of gingipains & P. gingivalis & Mishra et al., 2018 \\
\hline & RprY & Lysine residue & Bacterial survival & P. gingivalis & Li et al., 2018 \\
\hline & GtfB, GtfC, GtfD, & Lysine residue & Biofilm formation & S. mutans & Lei et al., 2020 \\
\hline \multicolumn{6}{|l|}{ Protein glycosylation } \\
\hline \multirow[t]{2}{*}{ N-glycosylation } & GspA & Asn residue & Bacterial survival & A. oris & $\begin{array}{l}\text { Li et al., 2004; Dige } \\
\quad \text { et al., } 2009\end{array}$ \\
\hline & CpsA & Asn residue & CPS synthesis & S. agalactiae & Stefanovic et al., 2021 \\
\hline \multirow[t]{2}{*}{ O-glycosylation } & LPS & $\begin{array}{l}\text { Ser and/or Thr } \\
\text { residue }\end{array}$ & Collagen-binding activity & A. actinomycetemcomitans & $\begin{array}{l}\text { Schaffer and Messner, } \\
2017\end{array}$ \\
\hline & Cnm, WapA & $\begin{array}{l}\text { Ser and/or Thr } \\
\text { residue }\end{array}$ & Adhesion & S. mutans & $\begin{array}{l}\text { Aviles-Reyes et al., } \\
\qquad 2018\end{array}$ \\
\hline \multirow[t]{2}{*}{ Protein citrullination } & PPAD & Arg residue & Bacterial survival & P. gingivalis & Stobernack et al., 2016 \\
\hline & Rgp & Arg residue & Inflammatory responses & $P$. gingivalis & Konig et al., 2015 \\
\hline Protein succinylation & Fimbriae, RagB, PorR & Lysine residue & Inflammatory responses & P. gingivalis & Wu et al., 2019 \\
\hline $\begin{array}{l}\text { Protein } \\
\text { S-glutathionylation }\end{array}$ & $\mathrm{Tlp}$ & Cys41 & $\begin{array}{l}\text { Maintaining intracellular redox } \\
\text { homeostasis }\end{array}$ & S. mutans & Li et al., 2020 \\
\hline
\end{tabular}

a cytoplasmic RR. The former is autophosphorylated on the His residue, and the phosphoryl group is subsequently transferred to aspartate (Asp) residue of the response regulator receiver domain, or additional domains, such as DNA-binding or other effector domains, to translate the sensed signal in response to environmental stimuli.

Two-component systems identified in oral bacteria play a crucial role in responding to environmental stresses and regulating bacterial virulence factors. VicRK, a member of TCS in Streptococcus mutans, is associated with biofilm formation. VicK can regulate the phosphorylation of the regulator VicR upon signal-induced autophosphorylation. Wang et al. (2021) reported that proline at position 222 in the PAS domain of VicK is responsible for its phosphatase activity, and the $\mathrm{VicK}^{\mathrm{P} 222 \mathrm{~A}}$ mutation inhibited its phosphatase activity and subsequently attenuated the phosphorylation of VicR that regulated the expression of $g t f B / C$, reducing the biofilm formation and bacterial growth in $S$. mutans. In addition, competence stimulating peptides (CSPs) could activate the His kinase ComD, which phosphorylates the response regulator ComE, leading to gene expression and induction of competence. Thus, competent $S$. mutans can take up exogenous DNA to develop bacterial resistance to antibiotics (Shepherd et al., 2012). Additional TCSs identified in S. mutans include NsrRS and LcrRS. NsrRS and LcrRS individually regulate the expression of $n s r X$ and the ABC transporter $l c t F E G$, which are associated with 


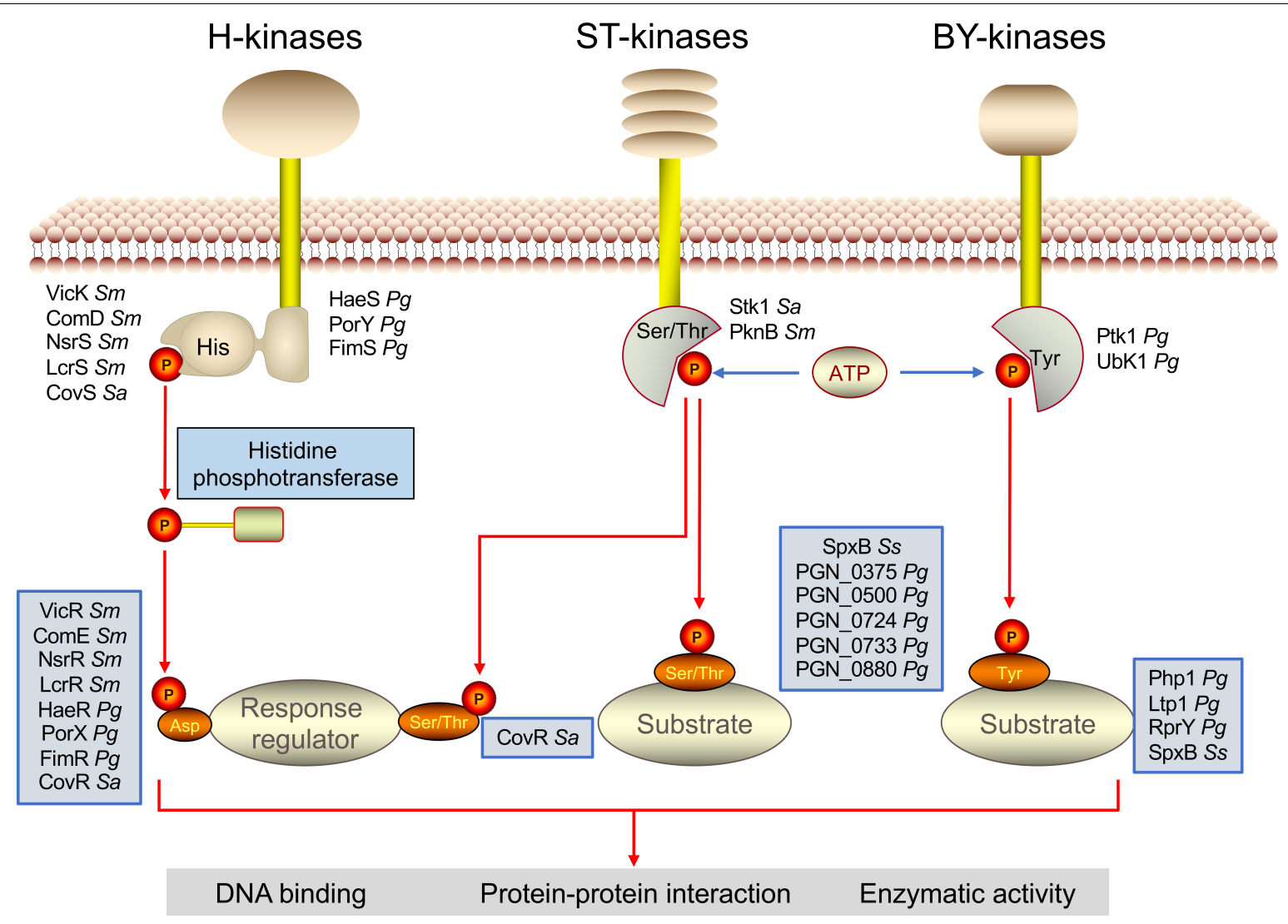

FIGURE 1 | Kinase-mediated protein phosphorylation in oral bacteria. Protein phosphorylation is involved in the kinase-catalyzed addition of phosphate to amino acid side chains of histidine (His), serine (Ser), threonine (Thr), and tyrosine (Tyr). The histidine phosphorylation in oral bacteria belongs to the two-component systems (TCS) composed of a membrane-located sensor His kinase and a cytoplasmic response regulator. The former comprises an extracellular sensory domain linked to a cytoplasmic domain required for catalysis and dimerization. The phosphate group is subsequently transferred from histidine to an aspartate residue of the latter. The activated response regulator mediates the physiological processes, mostly by regulating the transcription of some target genes. Bacterial Hanks-type ST-kinases are most commonly transmembrane proteins containing an extracellular domain binding to ligands and a cytosolic protein kinase domain. The kinase domain phosphorylates the serine and threonine residues of target substrates using the phosphate group of ATP as the donor. The protein tyrosine phosphorylation is catalyzed by the BY kinase family, which has thus far been found only in bacteria. BY kinases possess a transmembrane domain that functions not only as a kinase anchor but also as a sensor domain and an intracellular catalytic domain that can phosphorylate target proteins on tyrosine using the phosphate group as the donor. Pg for P. gingivalis, Sm for S. mutans, Sa for S. agalactiae, and Ss for S. sanguinis.

resistance against the lantibiotics nisin $\mathrm{A}$ and nukacin ISK-1, respectively (Kawada-Matsuo et al., 2013). In P. gingivalis, TCS HaeSR regulates numerous genes of iron uptake/acquisition that encode transporters and metabolic functions, which is vital for its survival. It was further demonstrated that ATP serves as the phosphodonor to phosphorylate the HaeS at the conserved histidine 226 residue (Huynh et al., 2010; Willett and Kirby, 2012). The PorXY has been demonstrated as the TCS and regulates the type IX secretion system in $P$. gingivalis (Kadowaki et al., 2016). In addition, TCS FimSR regulates the expression of fimA encoding the fimbrilin protein subunit of fimbriae, which is associated with biofilm formation and binding to other bacteria (Nishikawa et al., 2004).

\section{Serine/Threonine Phosphorylation}

The signaling systems composed of Hanks-type serine/threonine kinases (STKs) and phosphatases (STPs) also play an essential role in bacterial regulatory networks. STKs are either membrane or cytoplasmic proteins containing a catalytic domain involved in the phosphorylation of Ser/Thr residue and additional subdomains responsible for regulating their activities, binding to ligands, and protein-protein interactions. Similarly, STKs and STPs are engaged in responses to changing environmental conditions.

Although the systems are not involved in His phosphorylation, they can affect gene expression by directly phosphorylating TCS response regulators. It is reported that the STK Stk1 from S. agalactiae can phosphorylate the CovR response regulator at the Thr65 and then downregulate transcription of CAMP (Christie, Atkins Munch-Peterson) factor, a cytotoxin (Rajagopal et al., 2006; Lin et al., 2009). Differently, as a member of TCSs, the phosphorylation of CovR can be activated by HK CovS in S. agalactiae (Lamy et al., 2004; Jiang et al., 2005). It is reported that CovS activates the response regulator CovR by transphosphorylation at the aspartate residue 53 (D53) and subsequently the phosphorylated CovR upregulates the 
expression of CAMP (Rajagopal et al., 2006). In this case, CovR can be phosphorylated by $\mathrm{HK}$ CovS at D53 and also can be phosphorylated by STK Stk1 at Thr65. While the phosphorylation of CovR at Thr65 leads to inhibition of expression of CAMP, phosphorylation of CovR at D53 leads to activation. Apart from STK, cognate STPs Stp1 are engaged in this process. In addition, S. mutans contains one STK and one STP, referred to as $\mathrm{PknB}$ and $\mathrm{PppL}$, respectively. It has been reported that $\mathrm{PknB}$ regulates biofilm formation, bacteriocin production, and cell wall metabolism by modulating the activity of the TCS VicKR and ComDE in S. mutans (Banu et al., 2010; Zhu and Kreth, 2010). The Ser/Thr phosphorylation is engaged in other metabolic processes in oral bacteria in addition to regulating TCS. Streptococcal pyruvate oxidase (SpxB), a hydrogen peroxide-generating enzyme, was confirmed to have Thr phosphorylation. The mutated SpxB protein, including the substitution of Thr at positions 409, 415, and 508 with Asp, exhibited decreased solubility in vivo, contributing to bacterial growth, colony morphology, and hydrogen peroxide $\left(\mathrm{H}_{2} \mathrm{O}_{2}\right)$ production by mediating pyruvate conversion in Streptococcus sanguinis (Mu et al., 2021). Five phosphoproteins with Ser and/or Thr were identified in $P$. gingivalis, referred to as PGN_0375 (phosphoribulose/uridine kinase), PGN_0500 (methylmalonyl-CoA decarboxylase), PGN_0724 (NADdependent hydroxybutyrate dehydrogenase), PGN_0733 (alpha-glucan phosphorylase), and PGN_0880 (tryptophanase), using the phosphate-affinity chromatography and anti-Ser and -Thr antibodies. However, the specific functions and regulatory mechanisms should be further explored (Izumigawa et al., 2016).

\section{Tyrosine Phosphorylation}

Protein tyrosine phosphorylation is catalyzed by the tyrosine (BY) kinase family, which has thus far been identified only in bacteria (Mijakovic et al., 2016). A BY kinase possesses a transmembrane domain that can function not only as a kinase anchor but also as a sensor domain and an intracellular catalytic domain that binds ATP and transfers its phosphate to the hydroxyl unit of the tyrosine residue (Sun et al., 2020). The catalytic domain is defined by the canonical Walker A (P-loop) and $\mathrm{B}$ motif. In addition, some BY kinases also contain an additional Walker $\mathrm{A}^{\prime}$ motif. Walker $\mathrm{A}$ and $\mathrm{B}$ motifs are usually found in nucleotide-binding proteins; however, Walker $\mathrm{A}^{\prime}$ motif is found in certain kinase phosphorylation low-molecular-weight (LMW) substrates such as Ltp1 in P. gingivalis (Liu et al., 2017).

BY kinases have an essential role in the regulation of various signal transduction pathways via substrate phosphorylation. It has been reported that Ptk1, a BY kinase, can phosphorylate Php1 on Tyr residues 159 and 166, and Ltp1, which are responsible for exopolysaccharide production and regulating pathway constraining $P$. gingivalis-S. gordonii community development, and gingipain proteinases Kgp, RgpA, and RgpB phosphorylation, respectively (Jung et al., 2019). In vivo, loss of Php1 significantly reduces the ability of $P$. gingivalis to induce bone loss.

RprY, an orphan response regulator, could be phosphorylated on Tyr residue ( $\mathrm{Li}$ et al., 2018). The mutated phenylalanine (F) at Tyr41 (Y41F) diminished its affinity for the promoter region of $m f a 1$, responsible for the expression of genes encoding the type IX secretion system (T9SS) machinery and virulence factors secreted through the T9SS, including gingipain proteases and $P$. gingivalis-derived peptidylarginine deiminase (PPAD). Consistently, the RprY with a Y41F substitution impaired $P$. gingivalis virulence in a murine model of alveolar bone loss (Shen et al., 2020). Furthermore, a recent report showed that the Tyr phosphorylation of RprY is mediated by the Ubiquitous bacterial Kinase 1 (UbK1) (Perpich et al., 2021). In addition to Thr phosphorylation of SpxB, the Tyr phosphorylation at positions 409, 415, and 588 was also identified, with a possibly significant role in intraspecies and interspecies competition in S. sanguinis (Mu et al., 2021).

\section{Protein Acetylation}

Protein acetylation is another major regulatory PTM found ubiquitously in oral bacteria. The protein acetylation modifications are finely regulated by two distinct mechanisms: enzymatic and non-enzymatic acetylation (chemical acetylation; Figure 2). Enzymatic acetylation relies on protein acetyltransferase to transfer the acetyl group from Ac-CoA to lysine residue (Starai and Escalante-Semerena, 2004). Non-enzymatic acetylation requires acetyl phosphate (AcP) or Ac-CoA to serve as the donor of the acetyl group. Lysine acetylation is carried out covalently by adding an acetyl group to a lysine residue, changing the biochemical characteristics of target protein, such as their charge, stability, and/or conformation, and subsequently, altering the protein biological activity (Carabetta and Cristea, 2017; Sang et al., 2017).

Lysine acetylation has been shown to play an essential role in bacterial survival and virulence. Butler et al. (2015) identified 130 lysine-acetylated peptides from $92 P$. gingivalis proteins, including the three gingipains $\operatorname{RgpA}, \operatorname{RgpB}$, and Kgp in a $P$. gingivalis W40 acetylome study. In a previous report, the inactivation of $\operatorname{vim} A$, a putative acetyltransferase gene, resulted in the late-onset gingipain activity, compared with the wild-type W83, which is further explained in the following report. PG1842 (a functional VimA homolog) and VimA can acetylate pro-RgpB at residues K247 and K248, contributing to the activation/maturation of gingipains in $P$. gingivalis (Mishra et al., 2018).

In the acetylome of S. mutans, 973 acetylation sites were identified in 445 proteins, accounting for $22.7 \%$ of overall proteins, among which 617 acetylation sites were quantified in 302 proteins. Notably, the acetylation levels of glucosyltransferase B (GtfB), glucosyltransferase C (GtfC), and glucosyltransferase D (GtfD) in the biofilms decreased by 29,22 , and $37 \%$, respectively, compared to planktonic conditions (Lei et al., 2020). Gtfs are the critical virulence factors by which $S$. mutans can utilize sucrose to synthesize EPS, promoting biofilm formation, the initiating factor for caries. The results from the acetylome study indicate that the acetylation of Gtfs is an important mechanism of regulating their catalytic activity and subsequently the biofilm formation.

Lysine acetylation is reversed by deacetylases. Lysine deacetylases $\left(\mathrm{NAD}^{+}\right.$-dependent sirtuins; for example, CobB) can enzymatically remove the acetyl group from lysine side chains. Reports on the characterization of acetyltransferases and 


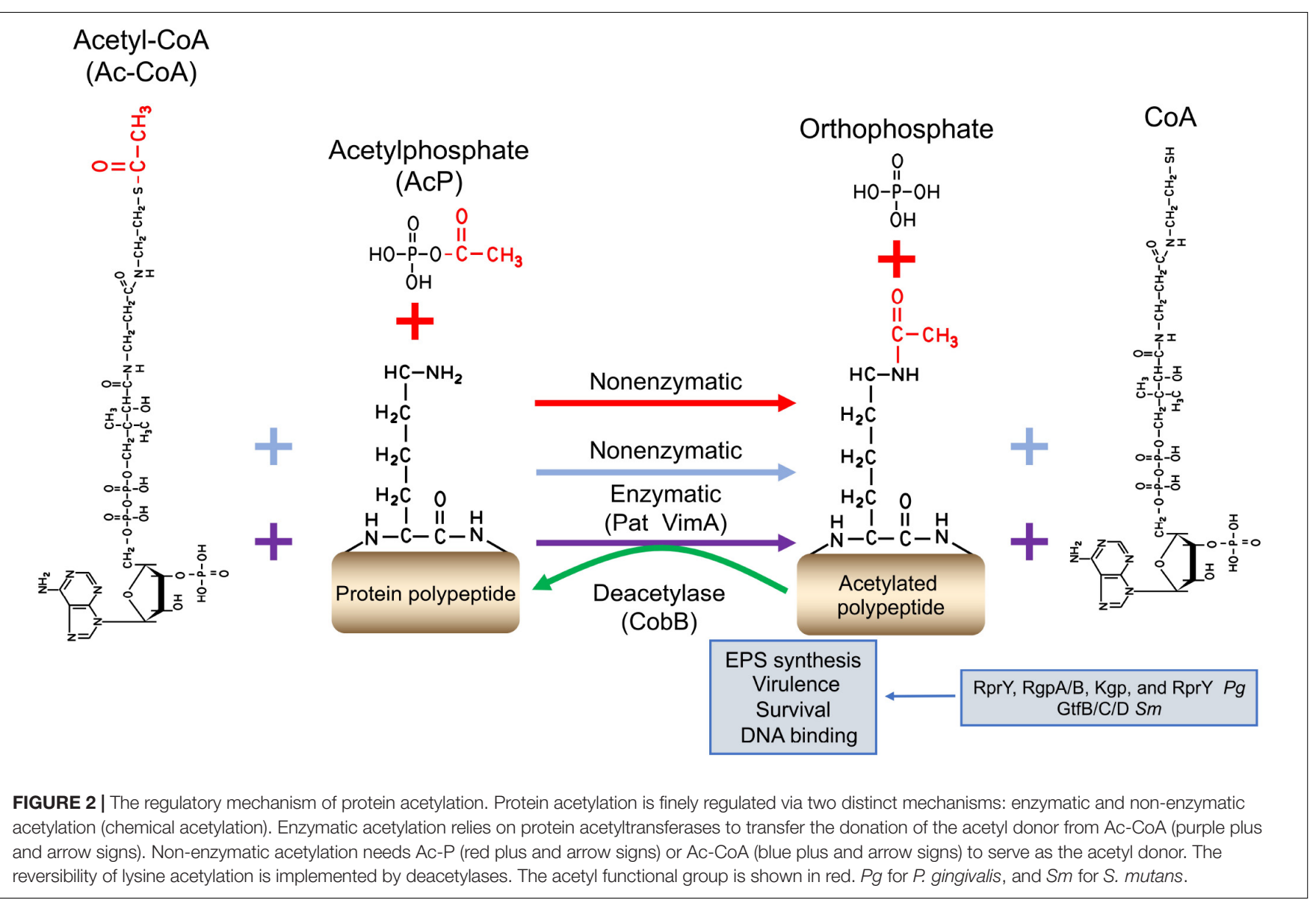

deacetylases emerge continuously, such as the acetyltransferase Pat and the deacetylase CobB from P. gingivalis. Li et al. (2018) reported that RprY could be modified by Pat with Ac-CoA serving as the acetyl group donor, and the acetylated RprY could be reversely deacetylated by CobB. Subsequently, the acetylation of RprY diminished its ability to bind the promoter DNA of nqrA (PGN_0115: $\mathrm{Na}^{+}$-translocating NADH-quinone reductase subunit $\mathrm{A}$ ), affecting bacterial survival in oxidative stress.

\section{Protein Glycosylation}

Protein glycosylation is considered one of the most abundant forms of PTMs and identified as a critical regulator in bacterial physiology, such as communication, adhesion, and virulence (Nothaft and Szymanski, 2010). It entails the covalent attachment of a glycan group to the amino acid residue to form glycoproteins (Figure 3). The glycan donors are phosphate-activated sugars linked to nucleotides (UDP-, GDP-, and ADP-) or dolicholrelated lipid groups. Protein glycosylation can be categorized into two major types, $\mathrm{N}$-linkages and $\mathrm{O}$-linkages, based on the glycosidic linkage; however, Cys-S-linked and Trp-C-linked glycoproteins are also known (Stepper et al., 2011; Yoshimoto et al., 2021). $N$-glycosylation binds glycans to the asparagine (Asn) residues, while O-glycosylation occurs at Ser or Thr residues. Protein glycosylation results from the modification of glycosidases and glycosyltransferases, whose activities depend on various bacterial processes and environmental stresses (Schaffer and Messner, 2017).

\section{$\mathrm{N}$-glycosylation}

$\mathrm{N}$-glycosylation generally contains a common pentasaccharide core with $\mathrm{N}$-acetyl glucosamine (GlcNac) serving as the attachment site to the Asn residue in the specific protein. $N$-glycosylated proteins are commonly found on bacterial surfaces, as exemplified by the LyrT-A-Psr (LCP) transferases in Actinomyces oris (Gosschalk et al., 2020). A. oris (formerly known as Actinomyces naeslundii), a pioneer of the oral cavity, is found in significant numbers in dental plaque, as well as on mucosal surfaces in adults (Ellen, 1976; Li et al., 2004; Dige et al., 2009). LCP transferase can glycosylate GspA that regulates sortase enzyme (SrtA) activity, vital for bacterial survival, and can arrest A. oris. In S. agalactiae, CpsA is covalently attached to GlcNac of the peptidoglycan (PGN) backbone and is involved in cell wall maintenance and capsular polysaccharide (CPSCPS) synthesis (Stefanovic et al., 2021). Although known for many years, the study of $\mathrm{N}$-glycosylation in oral bacteria is limited, necessitating further exploration.

\section{O-glycosylation}

O-glycosylation is usually connected to hydroxyl oxygen of Ser or Thr residues through $\mathrm{N}$-acetylgalactosamine (GalNac). The main types of $O$-glycosylation are cell-surface proteins, such as 


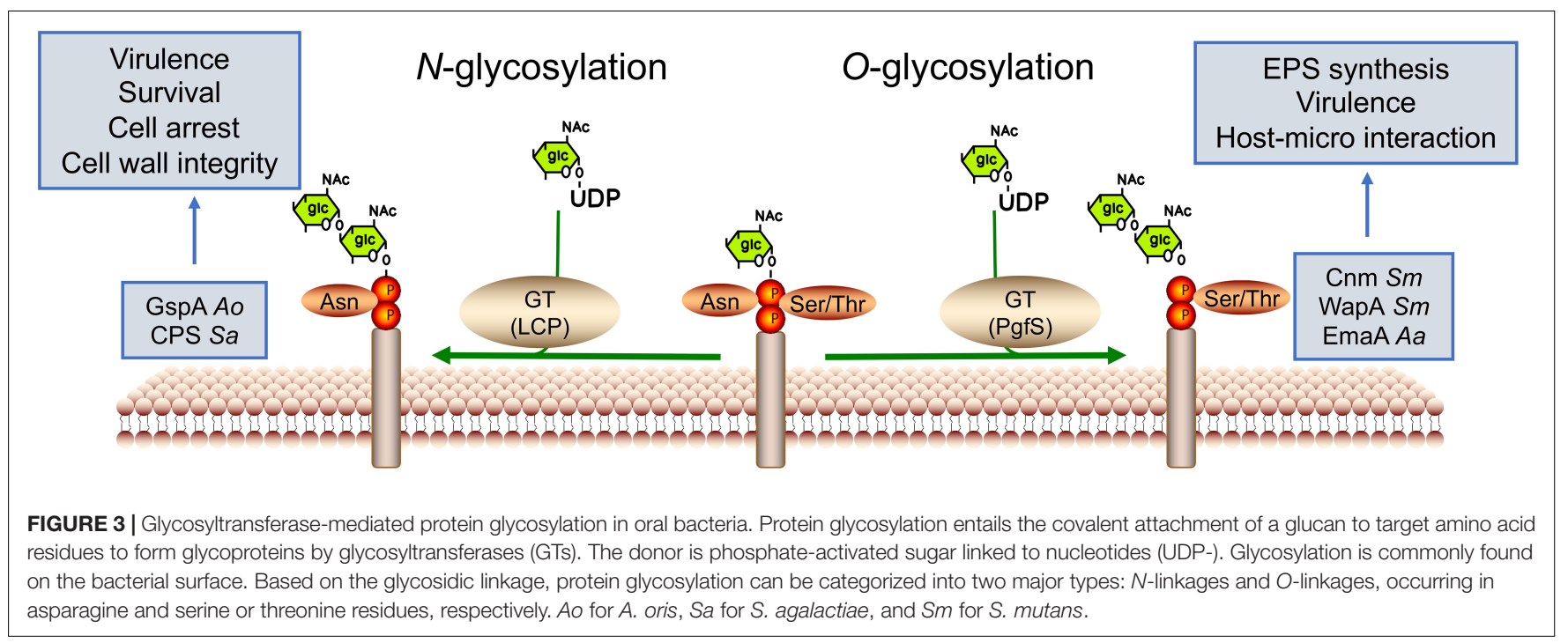

adhesins. The extracellular matrix protein adhesin A (EmaA), an adhesin, has been reported to be linked to the $O$-polysaccharide of the bacterium's LPS, maintaining collagen-binding activity in Aggregatibacter actinomycetemcomitans (Schaffer and Messner, 2017). The collagen- and laminin-binding protein (Cnm) and wall-associated protein A (WapA) could be glycosylated by glycosyltransferase PgfS, increasing their proteolytic stability and contributing to the adhesion of $S$. mutans to the host (Aviles-Reyes et al., 2018).

In addition to these O-GalNAc glycans, many proteins also have a single GlcNac covalently linked to Ser or Thr residues, similar to the glycosylation of SRR glycoproteins. SRR glycoproteins are a family of adhesins expressed by a wide range of oral bacteria, including S. gordonii, Streptococcus parasanguinis, S. agalactiae, and Lactobacillus species. The glycosylation of SRR glycoproteins contributes to colonization and virulence in various infections by binding to diverse host ligands (Chaze et al., 2014; Lee et al., 2014; Bleiziffer et al., 2017; Jiang et al., 2017). In S. gordonii, the SRR glycoprotein GspB can be glycosylated by glycosyltransferase GtfAB by transferring GlcNAc to Ser and Thr residues of the SRR domains, reducing its binding ability to human platelets. Moreover, GspB can be glycosylated by two additional Gtfs (Nss and Gly). After transferring Glc to GlcNAc by Nss, Gly transfers the second Glc residue, subsequently generating a trisaccharide (Seepersaud et al., 2020).

\section{Protein Citrullination}

Citrullination is an essential PTMs in which peptidylarginine is catalyzed to peptidyl-citrulline by PADs. The reaction is referred to as delamination due to the exchange of an imine for a carbonyl group (Tilvawala and Thompson, 2019). The PADcatalyzed reaction involves the hydrolysis of the guanidinium group of an arginine residue, leading to the loss of positive charge, generation of two hydrogen bond donors, and urea (Figure 4). Eventually, citrullination alters the charges of the substrate protein, possibly leading to significant changes in its structure and function (Wegner et al., 2010a). Although the existence of "decitrullinase" has been hypothesized, no such enzyme has been identified. The dysregulated PAD activity is observed in numerous inflammatory diseases including rheumatoid arthritis (RA), Alzheimer's disease, atherosclerosis, and periodontal diseases (PDs) (Vossenaar et al., 2003; Ishigami et al., 2005; Kinloch et al., 2008; Jones et al., 2009).

Interestingly, $P$. gingivalis secrets an analogous enzyme of PAD, known as PPAD. At present, $P$. gingivalis is the only known human pathogen producing cysteine-dependent PPAD. PPAD contains $\mathrm{N}$-terminal and C-terminal domains with different functions. $\mathrm{N}$-terminal domains are involved in substrate binding, protein-protein, and protein-nucleic acid interactions. C-terminal domains function as the catalytic domain, which can convert arginine residue to citrullinate. P. gingivalis secretes PPAD in a secreted soluble state or outer membrane vesicle (OMW)-bound state. Thus, PPAD causes aberrant citrullination that contributes to inflammatory responses in PD and can be an etiological agent of RA.

Gingipains, primarily with arginine residues (RgpA and RgpB), are associated with PPAD because fewer citrullinated proteins are detected in the Rgp deletion mutant than the parent strains (Wegner et al., 2010b). In addition, there is increasing evidence that PPAD-catalyzed protein citrullination depends on the function of Rgp proteases. Rgp proteases can cleave polypeptides at arginine residues, freeing a C-terminal arginine residue for PPAD to citrullinate. It is worth noting that Rgps with arginine residues can be citrullinated by PPAD (Stobernack et al., 2016). In addition, PPAD can be autocitrullinated on arginine residue 352 , reducing its activity and aid bacterial survival in periodontal pockets (Konig et al., 2015).

In addition to citrullinating bacterial proteins, PPAD has been shown to citrullinate specific human host proteins (Gabarrini et al., 2020). For example, PPAD can citrullinate the epidermal growth factor (EGF), blocking the recognition between the epithelium and the EGF signaling pathway molecules and impairing its ability to stimulate epidermal cell proliferation 


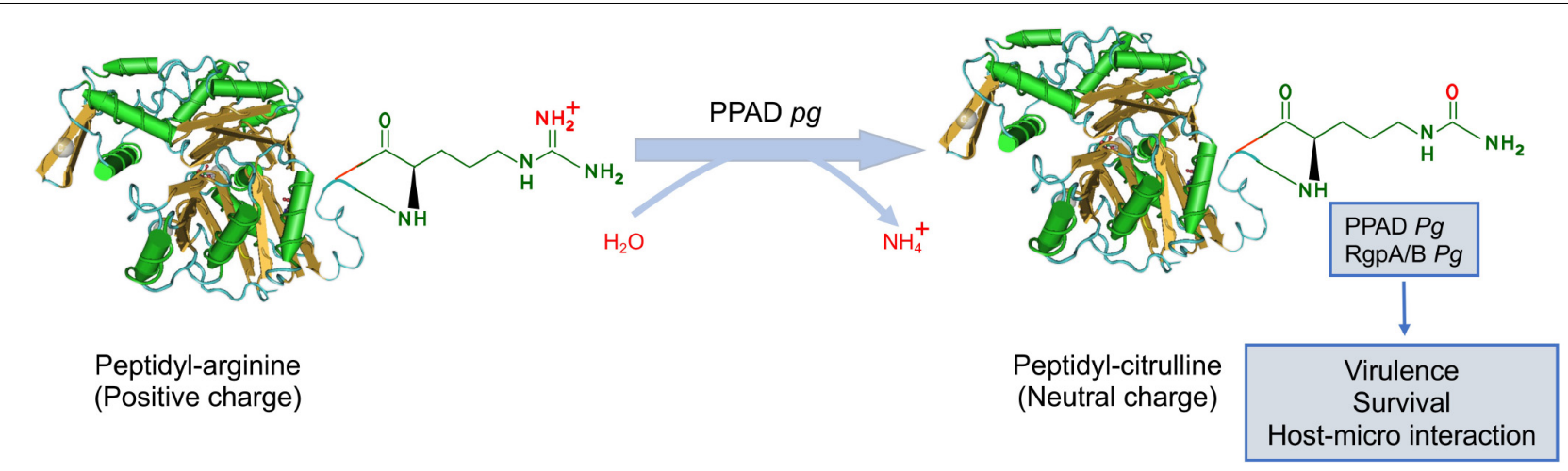

FIGURE 4 | The regulatory mechanism of protein citrullination. Protein citrullination is catalyzed by peptidylarginine deiminases (PADs), resulting in the conversion of arginine on the functional peptide to citrulline (defective protein), which leads to the loss of positive charge. At present, $P$. gingivalis is the only known pathogen that produces PAD, referred to as PPAD. PPAD not only citrullinates proteins in bacteria but also specific human host proteins by gaining access to the bloodstream. Pg for $P$. gingivalis.

and migration (Pyrc et al., 2013). In addition, the pore-forming leukotoxin (LtxA) secreted by A. actinomycetemcomitans is involved in host protein citrullination by activating endogenous PAD2 and PAD4 in neutrophils (Engstrom et al., 2018). The mechanisms and pathways responsible for human protein citrullination might indicate associations between oral bacteria and several systematic diseases (atherosclerosis, rheumatic arthritis, and Alzheimer's disease) (Olsen et al., 2018).

A recent analysis of $P$. gingivalis genomes of laboratory strains and clinical isolates unveiled a PPAD variant (PPAD-T2), with three amino-acid substitutions directly preceding the catalytic residue $\mathrm{H}^{236}\left(\mathrm{G}^{231} \mathrm{~N} / \mathrm{E}^{232} \mathrm{~T} / \mathrm{N}^{235} \mathrm{D}\right)$ compared with PPAD from the reference strain (PPAD-T1). Functionally, PPAD-T2 exhibits weaker substrate binding capability but higher catalytic rates than PPAD-T1. In addition, like PPAD-1, PPAD-T2 citrullinates arginine at the $\mathrm{C}$-terminal domain of the targeted protein (Bereta et al., 2019).

\section{Other Post-translational Modifications}

Additional PTMs have been identified and characterized in oral bacteria, with a vital role in bacterial physiological function and pathogenicity with the development of mass spectrometry. For example, a relatively recently discovered PTM is succinylation. Lysine succinylation has been defined as the transfer of a succinyl group to a lysine residue of the target protein, mediated either enzymatically or non-enzymatically with succinyl-CoA as the donor. Succinylation at lysine residues changes lysine's charge from +1 to -1 and introduces a relatively larger structure moiety, leading to a mass increase of $100 \mathrm{Da}$, expected to contribute to significant changes in protein structure and function. Over the past few years, lysine succinylation has been identified in a few oral bacterial species. An example is $P$. gingivalis. Several important virulence factors, gingipains, fimbriae, RagB, and PorR, occur in lysine succinylation (Wu et al., 2019). Furthermore, protein succinylation and acetylation were extensively overlapped in P. gingivalis, including PGN_0377, PGN_0457, PGN_0497, PGN_0723, PGN_0724, PGN_0725, PGN_1176, PGN_1178, PGN_1341, and PGN_1367, which play a crucial role in the ribosome and metabolic processes (Zeng et al., 2020).

$S$-glutathionylation is the specific PTM of protein cysteine thiols by incorporating tripeptide glutathione (GSH), in which cysteine thiol and glutathione are classified as low-molecularmass free (non-protein) thiols (Dalle-Donne et al., 2009). Thiols, organic sulfur derivatives (mercaptans), play a central role in coordinating the antioxidant signaling pathways. As a result, protein $S$-glutathionylation usually occurs in response to oxidative stress and prevents irreversible oxidation of cysteine residue, which is vital for maintaining intracellular redox homeostasis. A recent report showed that a thioredoxin-like protein (Tlp) is $S$-glutathionylated at Cys 41 residue, protecting $S$. mutans from reactive oxygen species (ROS) derived from hostile bacteria, e.g., S. sanguinis and S. gordonii (Li et al., 2020).

\section{CONCLUSION AND PERSPECTIVES}

As outlined in this review, PTMs are identified extensively in oral bacteria, with a profound influence on bacterial physiological processes and virulence regulation by altering the physicochemical nature of protein structure, activity, localization, and biomolecule interactions (Table 1). It is worth noting that protein modification machinery differs significantly in different bacterial species. For example, arginine phosphorylation is a protein degradation signal in the phylum Firmicutes, but so far, it has not been detected in other oral bacteria. Many oral bacterial PTMs depend on the metabolic status and specific products of metabolism. Acetylation is mainly catalyzed by acetyltransferase enzymatically and also by AcP non-enzymatically. However, more and more acetyl derivatives have been identified to participate in acetylation non-enzymatically in oral bacterial species, such as Ac-CoA and acetyladenylate, indicating that PTMs affect the intermediate products of primary metabolism, which reversibly regulate PTMs.

In general, a single protein can be modified by multiple PTMs either at the same or different sites. For example, 
lysine can be modified by a broad class of acylations, such as acetylation and succinylation. It has been demonstrated that both acetylation and succinylation modifications could occur in gingipains, fimbriae, RagB, and PorR, which are important virulence factors of $P$. gingivalis. The crosstalk between acetylation and phosphorylation was observed on RprY, and different modifications exert different biological functions. The acetylation of RprY reduces its ability to bind to the promoter of $n q r A$, while phosphorylation of RprY enhances its binding to the promoter of nqrA. Therefore, the crosstalk between different PTMs and their distinct and overlapping roles in regulating different physiological functions should be investigated further.

Many virulence- and survival-related proteins have been demonstrated to be modified in oral bacteria with recent advances in genomics and proteomics, and these studies suggest that PTMs play a critical role in bacterial virulence, survival, and pathogenicity. However, PTMs studies in oral bacterial species have mainly focused on a small number of species and strains, limiting the ability to explore the relationship between PTMs and bacterial functions and their regulatory mechanisms. For a complete understanding of the regulatory roles of PTMs, more studies will have to uncover the connection between the known modifications and their specific enzymes and vice versa. In conclusion,

\section{REFERENCES}

Aviles-Reyes, A., Freires, I. A., Besingi, R., Purushotham, S., Deivanayagam, C., Brady, L. J., et al. (2018). Characterization of the pgf operon involved in the posttranslational modification of Streptococcus mutans surface proteins. Sci. Rep. 8:4705. doi: 10.1038/s41598-018-23170-3

Banu, L. D., Conrads, G., Rehrauer, H., Hussain, H., Allan, E., and van der Ploeg, J. R. (2010). The Streptococcus mutans serine/threonine kinase. PknB, regulates competence development, bacteriocin production, and cell wall metabolism. Infect. Immun. 78, 2209-2220. doi: 10.1128/IAI.01167-09

Beck-Sickinger, A. G., and Mörl, K. (2006). Posttranslational modification of proteins. Expanding nature's inventory. By Christopher T. Walsh. Angew. Chem. Int. Ed. 45:1020. doi: 10.1002/anie.200585363

Bereta, G., Goulas, T., Madej, M., Bielecka, E., Sola, M., Potempa, J., et al. (2019). Structure, function, and inhibition of a genomic/clinical variant of Porphyromonas gingivalis peptidylarginine deiminase. Protein Sci. 28, 478-486. doi: 10.1002/pro.3571

Bleiziffer, I., Eikmeier, J., Pohlentz, G., McAulay, K., Xia, G., Hussain, M., et al. (2017). The plasmin-sensitive protein pls in methicillin-resistant Staphylococcus aureus (MRSA) is a glycoprotein. PLoS Pathog. 13:e1006110. doi: 10.1371/ journal.ppat. 1006110

Blod, C., Schlichting, N., Schülin, S., Suttkus, A., Peukert, N., Stingu, C. S., et al. (2018). The oral microbiome-the relevant reservoir for acute pediatric appendicitis? Int. J. Colorectal Dis. 33, 209-218. doi: 10.1007/s00384-0172948-8

Butler, C. A., Veith, P. D., Nieto, M. F., Dashper, S. G., and Reynolds, E. C. (2015). Lysine acetylation is a common post-translational modification of key metabolic pathway enzymes of the anaerobe Porphyromonas gingivalis. J. Proteomics 128, 352-364. doi: 10.1016/j.jprot.2015.08.015

Cain, J. A., Solis, N., and Cordwell, S. J. (2014). Beyond gene expression: the impact of protein post-translational modifications in bacteria. J. Proteomics 97 , 265-286. doi: 10.1016/j.jprot.2013.08.012

Carabetta, V. J., and Cristea, I. M. (2017). Regulation, function, and detection of protein acetylation in bacteria. J. Bacteriol. 199, e00107-e00117. doi: 10.1128/ JB.00107-17

Chaze, T., Guillot, A., Valot, B., Langella, O., Chamot-Rooke, J., Di Guilmi, A. M., et al. (2014). O-Glycosylation of the N-terminal region of the serine-rich identification and characterization of PTMs not only contributes to uncover their roles and underlying regulatory mechanisms in oral bacterial virulence, adaption and resistance, but will open new avenues for the treatment of oral infectious diseases.

\section{AUTHOR CONTRIBUTIONS}

QM and QZ drafted and wrote the manuscript. JZ and YuL critically reviewed the manuscript and improved it. YC, SY, $\mathrm{JH}$, YaL, and TG participated in manuscript correction. All authors gave final approval and agreed to be accountable for all aspects of the work.

\section{FUNDING}

This work was supported by grants from the National Natural Science Foundation of China (32170046 and 82170947), the Sichuan Science and Technology Program (2020YJ0296 and 2019YFH0025), and the Technological Innovation and Development Project of Chengdu Bureau of Science and Technology (2021-YF05-01980-SN).

adhesin Srr1 of Streptococcus agalactiae explored by mass spectrometry. Mol. Cell. Proteomics 13, 2168-2182. doi: 10.1074/mcp.M114.038075

Dalle-Donne, I., Rossi, R., Colombo, G., Giustarini, D., and Milzani, A. (2009). Protein S-glutathionylation: a regulatory device from bacteria to humans. Trends Biochem. Sci. 34, 85-96. doi: 10.1016/j.tibs.2008.11.002

Dige, I., Raarup, M. K., Nyengaard, J. R., Kilian, M., and Nyvad, B. (2009). Actinomyces naeslundii in initial dental biofilm formation. Microbiology (Reading) 155(Pt 7), 2116-2126. doi: 10.1099/mic.0.027706-0

Ellen, R. P. (1976). Establishment and distribution of Actinomyces viscosus and Actinomyces naeslundii in the human oral cavity. Infect. Immun. 14, 1119-1124. doi: 10.1128/iai.14.5.1119-1124.1976

Engstrom, M., Eriksson, K., Lee, L., Hermansson, M., Johansson, A., Nicholas, A. P., et al. (2018). Increased citrullination and expression of peptidylarginine deiminases independently of P. gingivalis and A. actinomycetemcomitans in gingival tissue of patients with periodontitis. J. Transl. Med. 16:214. doi: 10. 1186/s12967-018-1588-2

Gabarrini, G., Grasso, S., van Winkelhoff, A. J., and van Dijl, J. M. (2020). Gingimaps: protein localization in the oral pathogen Porphyromonas gingivalis. Microbiol. Mol. Biol. Rev. 84, e00032-e00119. doi: 10.1128/MMBR.00032-19

Gosschalk, J. E., Chang, C., Sue, C. K., Siegel, S. D., Wu, C., Kattke, M. D., et al. (2020). A cell-based screen in actinomyces oris to identify sortase inhibitors. Sci. Rep. 10:8520. doi: 10.1038/s41598-020-65256-x

Huynh, T. N., Noriega, C. E., and Stewart, V. (2010). Conserved mechanism for sensor phosphatase control of two-component signaling revealed in the nitrate sensor NarX. Proc. Natl. Acad. Sci. U.S.A. 107, 21140-21145. doi: 10.1073/pnas. 1013081107

Ishigami, A., Ohsawa, T., Hiratsuka, M., Taguchi, H., Kobayashi, S., Saito, Y., et al. (2005). Abnormal accumulation of citrullinated proteins catalyzed by peptidylarginine deiminase in hippocampal extracts from patients with Alzheimer's disease. J. Neurosci. Res. 80, 120-128. doi: 10.1002/jnr.20431

Izumigawa, M., Hasegawa, Y., Ikai, R., Horie, T., Inomata, M., Into, T., et al. (2016). Separation of novel phosphoproteins of Porphyromonas gingivalis using phosphate-affinity chromatography. Microbiol. Immunol. 60, 702-707. doi: 10.1111/1348-0421.12441

Jiang, S. M., Cieslewicz, M. J., Kasper, D. L., and Wessels, M. R. (2005). Regulation of virulence by a two-component system in group B streptococcus. J. Bacteriol. 187, 1105-1113. doi: 10.1128/JB.187.3.1105-1113.2005 
Jiang, Y. L., Jin, H., Yang, H. B., Zhao, R. L., Wang, S., Chen, Y., et al. (2017). Defining the enzymatic pathway for polymorphic O-glycosylation of the pneumococcal serine-rich repeat protein PsrP. J. Biol. Chem. 292, 6213-6224. doi: 10.1074/jbc.M116.770446

Jones, J. E., Causey, C. P., Knuckley, B., Slack-Noyes, J. L., and Thompson, P. R. (2009). Protein arginine deiminase 4 (PAD4): current understanding and future therapeutic potential. Curr. Opin. Drug Discov. Dev. 12, 616-627.

Jung, Y. J., Miller, D. P., Perpich, J. D., Fitzsimonds, Z. R., Shen, D., Ohshima, J., et al. (2019). Porphyromonas gingivalis tyrosine phosphatase php1 promotes community development and pathogenicity. mBio 10, e02004-e02019. doi: 10. 1128/mBio.02004- 19

Kadowaki, T., Yukitake, H., Naito, M., Sato, K., Kikuchi, Y., Kondo, Y., et al. (2016). A two-component system regulates gene expression of the type IX secretion component proteins via an ECF sigma factor. Sci. Rep. 6:23288. doi: $10.1038 /$ srep 23288

Kawada-Matsuo, M., Oogai, Y., Zendo, T., Nagao, J., Shibata, Y., Yamashita, Y., et al. (2013). Involvement of the novel two-component NsrRS and LcrRS systems in distinct resistance pathways against nisin A and nukacin ISK-1 in Streptococcus mutans. Appl. Environ. Microbiol. 79, 4751-4755. doi: 10.1128/ AEM.00780-13

Kinloch, A., Lundberg, K., Wait, R., Wegner, N., Lim, N. H., Zendman, A. J., et al. (2008). Synovial fluid is a site of citrullination of autoantigens in inflammatory arthritis. Arthritis Rheum. 58, 2287-2295. doi: 10.1002/art.23618

Konig, M. F., Paracha, A. S., Moni, M., Bingham, C. O. III, and Andrade, F. (2015). Defining the role of Porphyromonas gingivalis peptidylarginine deiminase (PPAD) in rheumatoid arthritis through the study of PPAD biology. Ann. Rheum. Dis. 74, 2054-2061. doi: 10.1136/annrheumdis-2014-205385

Lamy, M. C., Zouine, M., Fert, J., Vergassola, M., Couve, E., Pellegrini, E., et al. (2004). CovS/CovR of group B streptococcus: a two-component global regulatory system involved in virulence. Mol. Microbiol. 54, 1250-1268. doi: 10.1111/j.1365-2958.2004.04365.x

Lee, I. C., van, S. II, Tomita, S., Morsomme, P., Rolain, T., Hols, P., et al. (2014). GtfA and GtfB are both required for protein O-glycosylation in Lactobacillus plantarum. J. Bacteriol. 196, 1671-1682. doi: 10.1128/JB.01401-13

Lei, L., Zeng, J., Wang, L., Gong, T., Zheng, X., Qiu, W., et al. (2020). Quantitative acetylome analysis reveals involvement of glucosyltransferase acetylation inStreptococcus mutansbiofilm formation. Environ. Microbiol. Rep. 13, 86-97. doi: 10.1111/1758-2229.12907

Li, J., Helmerhorst, E. J., Leone, C. W., Troxler, R. F., Yaskell, T., Haffajee, A. D., et al. (2004). Identification of early microbial colonizers in human dental biofilm. J. Appl. Microbiol. 97, 1311-1318. doi: 10.1111/j.1365-2672.2004.0 2420.x

Li, Y., Krishnan, K., and Duncan, M. J. (2018). Post-translational regulation of a Porphyromonas gingivalis regulator. J. Oral Microbiol. 10:1487743. doi: 10 . 1080/20002297.2018.1487743

Li, Z., Zhang, C., Li, C., Zhou, J., Xu, X., Peng, X., et al. (2020). S-glutathionylation proteome profiling reveals a crucial role of a thioredoxin-like protein in interspecies competition and cariogenecity of Streptococcus mutans. PLoS Pathog. 16:e1008774. doi: 10.1371/journal.ppat.1008774

Lin, W. J., Walthers, D., Connelly, J. E., Burnside, K., Jewell, K. A., Kenney, L. J., et al. (2009). Threonine phosphorylation prevents promoter DNA binding of the Group B Streptococcus response regulator CovR. Mol. Microbiol. 71, 1477-1495. doi: 10.1111/j.1365-2958.2009.06616.x

Lira-Junior, R., and Boström, E. A. (2018). Oral-gut connection: one step closer to an integrated view of the gastrointestinal tract? Mucosal. Immunol. 11, 316-318. doi: $10.1038 / \mathrm{mi} .2017 .116$

Liu, B., Faller, L. L., Klitgord, N., Mazumdar, V., Ghodsi, M., Sommer, D. D., et al. (2012). Deep sequencing of the oral microbiome reveals signatures of periodontal disease. PLoS One 7:e37919. doi: 10.1371/journal.pone.0037919

Liu, C., Miller, D. P., Wang, Y., Merchant, M., and Lamont, R. J. (2017). Structurefunction aspects of the Porphyromonas gingivalis tyrosine kinase Ptk1. Mol. Oral Microbiol. 32, 314-323. doi: 10.1111/omi.12173

Loi, V. V., Rossius, M., and Antelmann, H. (2015). Redox regulation by reversible protein S-thiolation in bacteria. Front. Microbiol. 6:187. doi: 10.3389/fmicb. 2015.00187

Mijakovic, I., Grangeasse, C., and Turgay, K. (2016). Exploring the diversity of protein modifications: special bacterial phosphorylation systems. FEMS Microbiol. Rev. 40, 398-417. doi: 10.1093/femsre/fuw003
Mishra, A., Roy, F., Dou, Y., Zhang, K., Tang, H., and Fletcher, H. M. (2018). Role of acetyltransferase PG1842 in gingipain biogenesis in Porphyromonas gingivalis. J. Bacteriol. 200, e00385-e00418. doi: 10.1128/jb.00385-18

Mu, R., Anderson, D., Merritt, J., Wu, H., and Kreth, J. (2021). Post-translational modification of Streptococcus sanguinis SpxB influences protein solubility and H2 O2 production. Mol. Oral Microbiol. 36, 267-277. doi: 10.1111/omi.12348

Nishikawa, K., Yoshimura, F., and Duncan, M. J. (2004). A regulation cascade controls expression of Porphyromonas gingivalis fimbriae via the FimR response regulator. Mol. Microbiol. 54, 546-560. doi: 10.1111/j.1365-2958.2004.04291.x

Nothaft, H., and Szymanski, C. M. (2010). Protein glycosylation in bacteria: sweeter than ever. Nat. Rev. Microbiol. 8, 765-778. doi: 10.1038/nrmicro2383

Olsen, I., Singhrao, S. K., and Potempa, J. (2018). Citrullination as a plausible link to periodontitis, rheumatoid arthritis, atherosclerosis and Alzheimer's disease. J. Oral Microbiol. 10:1487742. doi: 10.1080/20002297.2018.1487742

Perpich, J. D., Yakoumatos, L., Johns, P., Stocke, K. S., Fitzsimonds, Z. R., Wilkey, D. W., et al. (2021). Identification and characterization of a UbK family kinase in Porphyromonas gingivalis that phosphorylates the RprY response regulator. Mol. Oral Microbiol. 36, 258-266. doi: 10.1111/omi.12347

Philip, N., Suneja, B., and Walsh, L. J. (2018). Ecological approaches to dental caries prevention: paradigm shift or shibboleth? Caries Res. 52, 153-165. doi: 10.1159/000484985

Pyrc, K., Milewska, A., Kantyka, T., Sroka, A., Maresz, K., Koziel, J., et al. (2013). Inactivation of epidermal growth factor by Porphyromonas gingivalis as a potential mechanism for periodontal tissue damage. Infect. Immun. 81, 55-64. doi: 10.1128/IAI.00830-12

Rajagopal, L., Vo, A., Silvestroni, A., and Rubens, C. E. (2006). Regulation of cytotoxin expression by converging eukaryotic-type and two-component signalling mechanisms in Streptococcus agalactiae. Mol. Microbiol. 62, 941-957. doi: 10.1111/j.1365-2958.2006.05431.x

Reddy, R. M., Weir, W. B., Barnett, S., Heiden, B. T., Orringer, M. B., Lin, J., et al. (2018). Increased variance in oral and gastric microbiome correlates with esophagectomy anastomotic leak. Ann. Thorac. Surg. 105, 865-870. doi: 10.1016/j.athoracsur.2017.08.061

Saikaly, S. K., Saikaly, T. S., and Saikaly, L. E. (2018). Recurrent aphthous ulceration: a review of potential causes and novel treatments. J. Dermatolog. Treat. 29, 542-552. doi: 10.1080/09546634.2017.1422079

Sang, Y., Ren, J., Qin, R., Liu, S., Cui, Z., Cheng, S., et al. (2017). Acetylation regulating protein stability and DNA-binding ability of HilD, thus Modulating Salmonella typhimurium virulence. J. Infect. Dis. 216, 1018-1026. doi: 10.1093/ infdis/jix102

Schaffer, C., and Messner, P. (2017). Emerging facets of prokaryotic glycosylation. FEMS Microbiol. Rev. 41, 49-91. doi: 10.1093/femsre/fuw036

Seepersaud, R., Anderson, A. C., Bensing, B. A., Choudhury, B. P., Clarke, A. J., and Sullam, P. M. (2020). O-acetylation controls the glycosylation of bacterial serine-rich repeat glycoproteins. J. Biol. Chem. 296:100249. doi: 10.1074/jbc. RA120.016116

Shen, D., Perpich, J. D., Stocke, K. S., Yakoumatos, L., Fitzsimonds, Z. R., Liu, C., et al. (2020). Role of the RprY response regulator in P. gingivalis community development and virulence. Mol. Oral. Microbiol. 35, 231-239. doi: 10.1111/ omi. 12311

Shepherd, N. E., Harrison, R. S., and Fairlie, D. P. (2012). Targeting quorum sensing and competence stimulation for antimicrobial chemotherapy. Curr. Drug. Targets 13, 1348-1359. doi: 10.2174/138945012803530233

Starai, V. J., and Escalante-Semerena, J. C. (2004). Identification of the protein acetyltransferase (Pat) enzyme that acetylates acetyl-CoA synthetase in Salmonella enterica. J. Mol. Biol. 340, 1005-1012. doi: 10.1016/j.jmb.2004. 05.010

Stefanovic, C., Hager, F. F., and Schaffer, C. (2021). LytR-CpsA-Psr glycopolymer transferases: essential bricks in gram-positive bacterial cell wall assembly. Int. J. Mol. Sci. 22:908. doi: 10.3390/ijms22020908

Stepper, J., Shastri, S., Loo, T. S., Preston, J. C., Novak, P., Man, P., et al. (2011). Cysteine S-glycosylation, a new post-translational modification found in glycopeptide bacteriocins. FEBS Lett. 585, 645-650. doi: 10.1016/j.febslet.2011. 01.023

Stobernack, T., Glasner, C., Junker, S., Gabarrini, G., de Smit, M., de Jong, A., et al. (2016). Extracellular proteome and citrullinome of the oral pathogen Porphyromonas gingivalis. J. Proteome Res. 15, 4532-4543. doi: 10.1021/acs. jproteome.6b00634 
Sun, C. F., Li, Y. Q., and Mao, X. M. (2020). Regulation of protein post-translational modifications on metabolism of actinomycetes. Biomolecules 10:1122. doi: 10 . 3390/biom 10081122

Tilvawala, R., and Thompson, P. R. (2019). Peptidyl arginine deiminases: detection and functional analysis of protein citrullination. Curr. Opin. Struct. Biol. 59, 205-215. doi: 10.1016/j.sbi.2019.01.024

Vossenaar, E. R., Zendman, A. J., van Venrooij, W. J., and Pruijn, G. J. (2003). PAD, a growing family of citrullinating enzymes: genes, features and involvement in disease. Bioessays 25, 1106-1118. doi: 10.1002/bies.10357

Wagner, G. R., and Hirschey, M. D. (2014). Nonenzymatic protein acylation as a carbon stress regulated by sirtuin deacylases. Mol. Cell. 54, 5-16. doi: 10.1016/j. molcel.2014.03.027

Wagner, G. R., and Payne, R. M. (2013). Widespread and enzyme-independent NEacetylation and $\mathrm{N} \varepsilon$-succinylation of proteins in the chemical conditions of the mitochondrial matrix. J. Biol. Chem. 288, 29036-29045. doi: 10.1074/jbc.M113. 486753

Walsh, C. T., Garneau-Tsodikova, S., and Gatto, G. J. Jr. (2005). Protein posttranslational modifications: the chemistry of proteome diversifications. Angew. Chem. Int. Ed. Engl. 44, 7342-7372. doi: 10.1002/anie.2005 01023

Wang, S., Long, L., Yang, X., Qiu, Y., Tao, T., Peng, X., et al. (2021). Dissecting the role of vick phosphatase in aggregation and biofilm formation of Streptococcus mutans. J. Dent Res. 100, 631-638. doi: 10.1177/0022034520979798

Wegner, N., Lundberg, K., Kinloch, A., Fisher, B., Malmström, V., Feldmann, M., et al. (2010a). Autoimmunity to specific citrullinated proteins gives the first clues to the etiology of rheumatoid arthritis. Immunol. Rev. 233, 34-54. doi: 10.1111/j.0105-2896.2009.00850.x

Wegner, N., Wait, R., Sroka, A., Eick, S., Nguyen, K. A., Lundberg, K., et al. (2010b). Peptidylarginine deiminase from Porphyromonas gingivalis citrullinates human fibrinogen and alpha-enolase: implications for autoimmunity in rheumatoid arthritis. Arthritis Rheum. 62, 2662-2672. doi: 10.1002/art. 27552

Willett, J. W., and Kirby, J. R. (2012). Genetic and biochemical dissection of a HisKA domain identifies residues required exclusively for kinase and phosphatase activities. PLoS Genet. 8:e1003084. doi: 10.1371/journal.pgen. 1003084

Wu, L., Gong, T., Zhou, X., Zeng, J., Huang, R., Wu, Y., et al. (2019). Global analysis of lysine succinylome in the periodontal pathogen Porphyromonas gingivalis. Mol. Oral Microbiol. 34, 74-83. doi: 10.1111/omi.12255

Yoshimoto, S., Katayama, K., Suzuki, T., Dohmae, N., and Simizu, S. (2021). Regulation of N-glycosylation and secretion of Isthmin-1 by its C-mannosylation. Biochim. Biophys. Acta Gen. Subj. 1865:129840. doi: 10.1016/ j.bbagen.2020.129840

Zeng, J., Wu, L., Chen, Q., Wang, L., Qiu, W., Zheng, X., et al. (2020). Comprehensive profiling of protein lysine acetylation and its overlap with lysine succinylation in the Porphyromonas gingivalis fimbriated strain ATCC 33277. Mol. Oral Microbiol. 35, 240-250. doi: 10.1111/omi.12312

Zhu, L., and Kreth, J. (2010). Role of Streptococcus mutans eukaryotic-type serine/threonine protein kinase in interspecies interactions with Streptococcus sanguinis. Arch. Oral Biol. 55, 385-390. doi: 10.1016/j.archoralbio.2010.03.012

Conflict of Interest: The authors declare that the research was conducted in the absence of any commercial or financial relationships that could be construed as a potential conflict of interest.

Publisher's Note: All claims expressed in this article are solely those of the authors and do not necessarily represent those of their affiliated organizations, or those of the publisher, the editors and the reviewers. Any product that may be evaluated in this article, or claim that may be made by its manufacturer, is not guaranteed or endorsed by the publisher.

Copyright (c) 2021 Ma, Zhang, Chen, Yu, Huang, Liu, Gong, Li and Zou. This is an open-access article distributed under the terms of the Creative Commons Attribution License (CC BY). The use, distribution or reproduction in other forums is permitted, provided the original author(s) and the copyright owner(s) are credited and that the original publication in this journal is cited, in accordance with accepted academic practice. No use, distribution or reproduction is permitted which does not comply with these terms. 